\title{
Como acessar a literatura biomédica e cruzar o "divisor de águas digital" em Pneumologia
}

\author{
How to Access the Biomedical Literature and Bridge the "Digital Divide" in \\ Pulmonology
}

\author{
JOÃO CARLOS PROLLA
}

Em países pobres e subdesenvolvidos, incluindo o Brasil, os pneumologistas têm enormes dificuldades em prover assistência médica de bom nível, em manterem-se em dia e em fazer pesquisa. Um de seus principais problemas é o acesso à literatura médica e científica, com os custos altíssimos e crescentes das assinaturas das revistas biomédicas. A expressão "divisor de águas digital" ("digital divide" em inglês) refere-se ao abismo entre aqueles que podem usar efetivamente as novas tecnologias de informação, como a Internet e aqueles que não tem acesso, tanto dentro dos países como entre os mesmos. Neste trabalho, chamamos a atenção para algumas soluções para esta situação de pobreza em informação, que estão disponíveis na Internet, a baixo ou quase nenhum custo. Em especial o projeto SciELO Brasil, o portal Periódicos CAPES, o PubMed e o projeto HINARI da OMS são detalhados e explicados.

J Bras Pneumol 2004; 30(5) 467-73
In poor and underdeveloped countries, including Brazil, pulmonologists have great difficulty in maintaining a level of service that keeps pace with advances in current knowledge, in keeping up to date with newly developed techniques, and in pursuing research. Due to the rising costs of scientific journal subscriptions, one of the main problems is lack of access to the scientific literature. The term "digital divide" refers to the gap between those who can and those who cannot effectively use new information technology and tools such as the Internet to communicate within and between countries. In this report, we call attention to some solutions to this situation. In particular, the SciELO Project, the CAPES Periodicals portal and the HINARI project, as well as several others, provide access at little or no cost. These options are explained in detail herein.
Descritores: Digital divide. Acesso online. Internet. Bibliografia pneumológica.
Key words: Pulmonary Disease (Specialty), Internet. Bibliography. Resource Guides. 


\section{INTRODUÇÃOO}

Em países pobres e subdesenvolvidos, incluindo - Brasil, os pneumologistas têm enormes dificuldades em prover assistência médica de bom nível, em manterem-se em dia e em fazer pesquisa. Um de seus principais problemas é 0 acesso à literatura médica e científica, com os custos altíssimos e crescentes das assinaturas das revistas biomédicas. A expressão "divisor de águas digital" ("digital divide" em inglês) refere-se ao abismo entre aqueles que podem usar efetivamente as novas tecnologias de informação, como a Internet e aqueles que não tem acesso, tanto dentro dos países como entre os mesmos ${ }^{(1)}$.

Com um computador pessoal (PC) conectado à Internet, é possível construir uma espécie de biblioteca eletrônica, a um custo inicial de aproximadamente $R \$ 3.000,00$ (custo médio de um $\mathrm{PC}$ ) e a um custo mensal adicional de cerca de $\mathrm{R} \$$

40,00 para cobrir 0 acesso eletrônico, em geral por linha telefônica, de um servidor comercial.

Cerca de 2,5 milhões de artigos científicos (em mais de 24.000 periódicos, dos quais um pouco mais de $10 \%$ são revistas médicas) são publicados anualmente. Estes números gigantescos apontam para a necessidade de bancos de dados digitais indexados e referenciáveis. É interessante assinalar que há um movimento mundial para considerar o custo de ter os artigos científicos depositados em tais bancos de dados, como inseparável e parte integrante dos custos das publicações científicas, devendo ser coberto pelo grant da pesquisa ou pela instituição que originou a mesma(2). A Budapest Open Access Initiative - BOAI (www.soros.org/o penaccess/ ) tem um fórum de debates muito ativo deste aspecto e de muitos outros problemas correlatos. A BOAI é uma declaração de princípios e um plano de ação, com a finalidade de atingir "open access to peer-reviewed research articles in all academic fields and the preprints that precedem them" e foi formalmente lançada em 14 de fevereiro de 2002 em Budapeste. 0 milionário George Soros fez quatro dias depois uma doação de US $\$ 3,000,000.00$ para a BOAI, o que tem sido de inestimável valia para a iniciativa(www.infotoday.com/newsbreaks/ nb020218-1.htm).

Para cruzar a "Digital Divide" existem algumas rotas de acesso, a custo muito baixo ou até mesmo a custo zero, a partes importantes (infelizmente ainda muito limitadas) da literatura biomédica eletrônica, que passaremos a explorar no restante de nosso trabalho.
Siglas e abreviaturas utilizadas neste trabalho:

BOAl: Budapest Open Access Initiative

DOAJ : Directory of Open Access Journals

FAPESP: Fundação de Amparo à Pesquisa do Estado de São Paulo HCPA: Hospital de Clínicas de Porto Alegre

HINARI: Health Internetwork Access to Research Initiative ISI: Institute for Scientific Information

NDLTD: Digital Library of Theses and Dissertations

NML: National Libray of Medicine

OMS: Organização Mundial da Saúde

PC: computador pessoal

PLoS: The Public Library of Science

Para um acesso inteiramente grátis aos títulos e resumos de artigos, o portal de serviços PubM ed pode ser acessado via Entrez, sistema de busca e acesso baseado nos textos, usado pelo NCBI para seus grandes bancos de dados, PubMed, Nucleotide and Protein Sequences, Protein Structures, Complete Genomes, Taxonomy (www.ncbi.nlm.nih.gov/PubM ed/). É uma ferramenta extremamente útil para explorar a literatura biomédica corrente de qualquer tópico ou assunto. A partir do resultado da busca, poderemos selecionar os artigos mais relevantes e então buscar os textos completos. É certamente uma das razões identificadas para o decréscimo no uso do material impresso na maioria das bibliotecas médicas ${ }^{(3)}$.

Para um acesso aberto, ver definição na referência(4), ou de baixo custo aos textos completos dos artigos científicos, existem alguns portais eletrônicos, entretanto a maioria deles é bastante limitada no número dos periódicos disponíveis, como PLoS ou BioM ed Central, ou no tipo de usuário aceito: HINARI.

The Public Library of Science (PLoS) (www.publiclibraryofscience.org/)

É uma organização sem fins lucrativos de médicos e cientistas comprometidos em tornar a literatura médica e científica mundial um recurso público livre e gratuito. 0 texto a seguir é reproduzido na íntegra a partir da website da organização:

"The internet and electronic publishing enable the creation of public libraries of science containing the full text and data of any published research article, available free of charge to anyone, anywhere in the world. Immediate unrestricted access to scientific ideas, methods, results, and conclusions will speed the progress of science and medicine, and will more directly bring the benefits of research to the public.

To realize this potential, a new business model for scientific publishing is required that treats the 
costs of publication as the final integral step of the funding of a research project. To demonstrate that this publishing model will be successful for the publication of the very best research, PLOS will publish its own journals. PLOS Biology launched its first issue on October 13, 2003, in print and online. PLOS Medicine will follow in 2004.

PLOS is working with scientists, their societies, funding agencies, and other publishers to pursue our broader goal of ensuring an open-access home for every published article and to develop tools to make the literature useful to scientists and the public."

Ao contrário da BOAI, que prega 0 acesso livre desde a data da publicação do artigo de pesquisa, a PLOS aceita uma moratória de 6 meses até dois anos depois, embora propague que 0 ideal seja 0 acesso livre imediato.

\section{Health Internetwork Access to Research Initiative (HINARI) \\ (www.healthinternetwork.org)}

Lançado pelo Secretário geral da ONU em setembro de 2000 e mantido pela Organização Mundial da Saúde (OMS), o HINARI ou Health InterNetwork tem produzido parcerias entre agências governamentais e privadas, sob o princípio do acesso equânime à informação em saúde pelos sistemas de saúde dos países contemplados. Os elementos básicos do projeto são conteúdo, conectividade com a Internet e capacidade de construção progressiva.

Mais de 2000 periódicos estão disponíveis no portal HINARI. Essa coleção foi feita inicialmente pela parceria da OM S com as seis maiores empresas de publicação biomédica: Blackwell, Elsevier Science, Harcourt Worldwide STM Group, Wolters Kluwer International Health \& Science, Springer Verlag, e J ohn Wiley. Landes Bioscience e mais 22 empresas reuniram-se posteriormente ao grupo. Foi descrita pelo Diretor Geral da OMS, Dr Gro Harlem Brundtland, como "perhaps the biggest step ever taken towards reducing the health information gap between rich and poor countries."

HINARI tem atualmente 99 países em desenvolvimento em sua lista de beneficiários. Infelizmente o Brasil não pode ser incluído por ter renda bruta per capita acima do limite estabelecido pela ONU, baseado em dados do Banco Mundial de 1998. Instituições de países com renda bruta per capita abaixo de US $\$ 1,000.00$ tem acesso grátis, e instituições de países com renda per capita entre US\$1,000.00 e
US\$ 3,000.00 tem acesso a preços reduzidos.

Em editorial de fevereiro de 2003 do British Medical Journal, Richard Smith avaliou os resultados dos primeiros dois anos de uso do HINARI como abaixo de ótimo(5).

Outros portais de acesso livre

Os portais abaixo relacionados permitem acesso livre a muitas coleções de periódicos ou de publicações biomédicas como teses, capítulos de livros, etc.

BioM ed Central

(www.biomedcentral.com)

Free Medical J ournals

(www.freemedicaljournals.com)

Free Books for Doctors

(www.freebooks4doctors)

PubMed Central

(www.pubmedcentral.nih.gov/

SciELO (www.scielo.org)

Periódicos CAPES

(www.periodicos.capes.gov.br/)

Directory of Open Access J ournals DOAJ

(www.doaj.org/)

SPARC (www.arl.org/sparc/)

PathMax (www.pathmax.com/)

\section{Biomed Central}

Os artigos de pesquisa científica publicados nos periódicos do BioM ed Central são "open access". Estão imediata e permanentemente disponíveis na Internet sem nenhum custo. Alguns periódicos exigem uma assinatura institucional ou pessoal para acessar outros tipos de artigos, como revisões ou relatórios.

Para os pneumologistas não há periódico clínico disponível, mas acreditamos que os periódicos de ciências básicas seguintes podem ter interesse, mas é claro que cada um pode acessar a lista completa dos periódicos:
BMC Cancer
BMC Cell Biology
BMC Clinical Pathology
BMC Molecular Biology
BMC Public Health
BMC Pulmonary Medicine
Cancer Cell International
Cell \& Chromosome
J ournal of Biology
J ournal of Carcinogenesis
Molecular Cancer 
Free Medical J ournals

O Free Medical Journals Site é dedicado à promoção do livre acesso aos periódicos médicos através da Internet. Atualmente existem 1.350 revistas médicas no portal, em geral com início de acesso retroativo aos anos 90.0 s periódicos da pneumologia mais importantes são os seguintes:

American Journal of Respiratory and Critical Care Medicine

Asthma Magazine

BMC Pulmonary Medicine - livre após um ano

Chest - livre após um ano

Enfermedades del Tórax

Euro TB

Indian Journal of Chest Diseases and Allied Sciences

Jornal de Pneumologia

Primary Care Respiratory J ournal

Pulmonary and CriticalCare Update

Pulmonary Perspectives

Respiratory Research

Revista Chilena de Enfermedades Respiratórias

Thorax - livre após um ano

Os periódicos fora da especialidade mais pertinentes são os seguintes:

BMJ - livre sem restrições

Circulation - livre após um ano

Current Allergy and Clinical Immunology

J ournal of Clinical Investigation

J ournal of Infectious Diseases - livre após dois anos

Molecular Biology of the Cell - livre após 2 meses da publicação

Molecular and Cellular Biology

New England Journal of Medicine - (Original Articles) - livre após 6 meses

Occupational Medicine - livre após dois anos

Radiology - livre após dois anos

Science - livre após um ano

PubMed Central

PubMed Central (PMC) é o arquivo digital da literatura das ciências da vida pertencente à U.S. National Library of Medicine (NLM). 0 acesso é livre eilimitado. A NLM está digitalizando todos os números antigos dos periódicos de sua lista, para colocá-los à disposição no PMC. No portal podemos acessar:
Respiratory Research

BMC Pulmonary Medicine

SCIELO

As informações abaixo foram retiradas do portal SciELO e explicam como funciona este modelo de acesso à informação técnico-científica.

"O acesso adequado e atualizado à informação técnico-científica é essencial para 0 desenvolvimento econômico e social, especialmente para apoiar os processos de tomada de decisão na planificação, formulação e aplicação de políticas públicas ou para apoiar o desenvolvimento e a prática profissional. 0 resultado da pesquisa científica é comunicado e validado principalmente através da publicação em periódicos científicos. Esse processo é válido para os países desenvolvidos ou em desenvolvimento. Ainda assim, os periódicos científicos dos países em desenvolvimento enfrentam graves barreiras de distribuição e disseminação, o que limita 0 acesso e o uso da informação científica gerada localmente.

SCIELO - Scientific Electronic Library Online (Biblioteca Científica Eletrônica em Linha) é um modelo para a publicação eletrônica cooperativa de periódicos científicos na Internet. Especialmente desenvolvido para responder às necessidades da comunicação científica nos países em desenvolvimento e particularmente na América Latina e Caribe, o modelo proporciona uma solução eficiente para assegurar a visibilidade e 0 acesso universal à sua literatura científica, contribuindo para a superação do fenômeno conhecido como "ciência perdida". O M odelo SciELO contém ainda procedimentos integrados para medir o uso e o impacto dos periódicos científicos.

O Modelo SciELO é o produto da cooperação entre a FAPESP (www.fapesp.br) - Fundação de Amparo à Pesquisa do Estado de São Paulo, BIREME (www.bireme.br) - Centro LatinoAmericano e do Caribe de Informação em Ciências da Saúde, instituições nacionais e internacionais relacionadas com a comunicação científica e editores científicos. Um projeto piloto, envolvendo 10 periódicos brasileiros de diferentes áreas do conhecimento, foi desenvolvido com êxito entre Março de 1997 e Maio de 1998, com o desenvolvimento e a avaliação de uma metodologia adequada para a publicação eletrônica na Internet. Desde Junho de 1998 o projeto opera 
regularmente, incorporando novos títulos de periódicos e expandindo sua operação para outros países. A partir de 2002, o Projeto conta com 0 apoio do CNPq (www.cnpq.br) - Conselho Nacional de Desenvolvimento Científico e Tecnológico.

O Modelo SciELO contém três componentes.

0 primeiro componente é a Metodologia SciELO, que permite a publicação eletrônica de edições completas de periódicos científicos, a organização de bases de dados bibliográficas e de textos completos, a recuperação de textos por seu conteúdo, a preservação de arquivos el etrônicos e a produção de indicadores estatísticos de uso e impacto da literatura científica. A Metodologia também inclui critérios de avaliação de revistas, baseado nos padrões internacionais de comunicação científica. Os textos completos são enriquecidos dinamicamente com links de hipertexto com bases de dados nacionais e internacionais, como por exemplo, LILACS e MEDLINE.

O segundo componente do Modelo SciELO é a aplicação da Metodologia SciELO na operação de websites de coleções de revistas eletrônicas. 0 Modelo SciELO favorece a operação de sites nacionais e também de sites temáticos. A aplicação pioneira é o site SciELO Brasil (www.scielo.br). Também estão em operação aplicações no Chile (www.scielo.cl) e em Cuba (www.scielo.sld.cu). Outros países estão avaliando ou recebendo treinamento da Metodologia SciELO. SciELO Saúde Pública (www.scielosp.org), uma biblioteca temática regional que cobre a área de Saúde Pública com periódicos científicos da América Latina e Espanha, foi inaugurada em Dezembro de 1999. Um portal para integrar e prover acesso à rede de sites SciELO está em operação em www.scielo.org.

0 terceiro componente do Modelo é o desenvolvimento de alianças entre os atores nacionais e internacionais da comunicação científica - autores, editores, instituições científico-tecnológicas, agências de financiamento, universidades, bibliotecas, centros de informação científica e tecnológica etc, com o objetivo de disseminar, aperfeiçoar e atualizar - Modelo SciELO. A operação da rede SciELO baseia-se fortemente em infra-estruturas nacionais, o que contribui para garantir sua futura sustentabilidade."
Para a pneumologia brasileira foi uma grande vitória a inclusão do Jornal de Pneumologia no portal Scielo(6), com isto a produção científica brasileira nesta especialidade deixou de fazer parte da chamada "ciência escondida ou perdida"(7).

\section{Portal CAPES Periódicos}

(www.periodicos.capes.gov.br/)

Pesquisadores brasileiros trabalhando em mais de cem universidades, institutos de pesquisa e hospitais federais autorizados podem acessar um grande número de periódicos internacionais através do portal CAPES Periódicos. As renovações e novas inclusões tornarão disponível, em 2004, um total de 4.585 periódicos com texto completo pago (acréscimo de cerca de 30\% sobre 2003), incluindo como adições as grandes empresas Kluwer, Oxford e Springer-Verlag, afora naturalmente os periódicos de acesso gratuito. Mas não podemos esquecer que as assinaturas eletrônicas custam milhões de dólares, que poderiam ser empregados em auxílios à pesquisa, equipamentos e grants, se esses periódicos tivessem "open access".

Pesquisadores argentinos autorizados podem acessar os mesmos periódicos através de sua Biblioteca Electrónica de Ciencia y Tecnologia (biblioteca.secyt.gov.ar/). Ambos portais também dão acesso ao ISI Web of Knowledge, do 'Institute for Scientific Information", onde qualquer cientista pode identificar quem, onde e quando houve uma citação de qualquer artigo da literatura científica moderna, nos periódicos indexados por aquela empresa, é importante esclarecer.

Os principais periódicos disponíveis são:

Ann Thorac Surg

Chest

DOAJ (www.doaj.org/)

Directory of Open Access J ournals. Este serviço da Lund University Libraries, abrange uma coleção de periódicos de livre acesso, textos completos e com qualidade controlada. É do seu intento abranger todas as áreas e idiomas de publicação. Pertence ao grupo SPARC (ver adiante). Atualmente estão no diretório 551 periódicos. O BMC Pulmonary Medicine está no portal (ver BioMed Central acima).

Digital Library of Theses and Dissertations NDLTD (www.ndltd.org/)

É um repositório digital de teses e dissertações 
mantido pela Virginia Tech University (USA). Existem inúmeros exemplares de pesquisa em pneumologia e ciências afins disponíveis. 0 site tem uma ferramenta de busca muito eficiente. Vale a pena visitar e navegar em busca de informação e sugestões para projetos de pesquisa. Seria interessante que todos os programas de pósgraduação brasileiros criassem um banco de dados semelhante, talvez no portal SciELO.

\section{SPARC (www.arl.org/sparc/)}

SPARC é uma aliança de universidades, bibliotecas e institutos de pesquisas, construída em resposta às disfunções de mercado na comunicação acadêmica e de pesquisa. É notório que essas disfunções, em especial o altíssimo preço das assinaturas, tem reduzido a disseminação das pesquisas e tornado muitas bibliotecas pouco eficientes. SPARC serve como um catalisador de ação, ajudando a criar sistemas para expandir a disseminação da informação e seu uso num ambiente digital interligado, responsivo às necessidades dos pesquisadores e professores. Exemplos: DOAJ (www.doaj.org/) e FIGARO (www.figaro-europe.net/)

PathM ax (www.pathmax.com/)

PathMax é um grande meta-índice de patologia, com mais de 3.200 links, quase todas as disciplinas da patologia na Internet e revisada como digna de figurar no índice. É muito fácil de usar, e mantido up to date pelo Dr Shawn E. Cowper, M.D. e seu corpo de assistentes voluntários, que cuidam de tópicos específicos. Há um grande número de sites com informações de patologia respiratória que podem ser acessados. 0 diretório de Citopatologia está sob nossos cuidados e o de Microscopia por nosso colaborador Dr Vinicius Duval da Silva. Dentro do diretório de patologia cirúrgica, há um sub-diretório de patologia pulmonar e torácica, sob os cuidados do Dr. M. McCormick, com links muito úteis, desde um site que avalia os resultados de um lavado broncoalveolar, vários testes de auto-avaliação em patologia pulmonar, ou até mesmo as últimas informações sobre a SARS pelo CDC de Atlanta. Em nossas aulas de patologia pulmonar usamos com grande freqüência esses recursos.

o trecho abaixo foi extraído de sua página inicial explicativa de sua missão:
"PathM ax and its mirrors were created to allow pathologists, residents, and other medical professionals to utilize the resources of the World Wide Web speedily and effectively. Each link is personally visited by the author and individual topic link editors and evaluated for its potential usefulness to the target audience. (...) Those wishing to link to this site need not obtain permission. There is no charge for PathMax, nor will there ever be. (...)"

0 site tem um espelho no CAP Superlinks (www.cap.org/superlinks/).

PTOLEMY (www.utoronto.ca/ois/myweb9/ index.htm)

Ptolemy foi idealizado pelo cirurgião da University of Toronto, Dr. M assey Beveridge (8). É engenhosamente simples. Em vez de dar acesso eletrônico a escolas médicas ou institutos de pesquisa, Ptolemy deu o mesmo a 100 médicos em prática efetiva - neste caso, todos da África Oriental. Para tornar isso possível, a University of Toronto nomeou esses médicos "research affiliates" de seu Office of International Surgery e montou servidores proxy em Toronto para acomodá-los. A universidade já tinha convênios de licença de uso para seus research affiliates. Imediatamente, isto deu aos doutores africanos acesso completo às assinaturas eletrônicas da biblioteca da University of Toronto, a maior biblioteca médica do Canadá e a terceira na América do Norte. Isso inclui o texto completo de 20.000 periódicos, centenas de livros médicos e muitos outros recursos eletrônicos.

A beleza do projeto é que é modulável e poderá ser expandido conforme demanda orientada e monitorada, podendo ser adotado por outras universidades do primeiro mundo, em socorro dos médicos do terceiro mundo.

\section{CONCLUSÃO}

Embora ainda limitada, existe uma grande quantidade de periódicos médicos que podem ser acessados com custo módico por nossos pneumologistas, em especial aqueles que tem acesso ao portal Periódicos CAPES. Ainda falta um mecanismo mais abrangente e com certeza 
de sustentabilidade a longo prazo para os pneumologistas sem acesso ao portal CAPES. A exclusão do Brasil no projeto HINARI é uma infelicidade, no caso uma ironia do destino, pois nossa renda per capita nos coloca além do seu uso. Nossa comunidade deveria trabalhar mais efetivamente para que todos nós possamos cruzar o divisor de águas digital. Na minha área principal de atuação, a citopatologia, aderi ao corpo editorial de uma revista eletrônica que deverá ser lançada em 2004. Um grande passo seria insistir que toda pesquisa fomentada e paga pelo poder público seja tornada acessível online de modo amplo e sem custo para os usuários.

\section{REFERÊNCIAS}

1. Warschauer M. Demystifying the digital divide. Sci Am. 2003;289:34-9.

2. Prosser DC. "Author pays" publishing model: NHS authors now enjoy free open access publication. BMJ. 2003;327:53-4.

3. De Groote SL, Dorsch JL. Online journals: impact on print journal usage. Bull Med Libr Assoc. 2001;89:372-8.

4. Till JE. Success factors for open access. J Med Internet Res. 2003;5:1.

5. Smith R. Closing the digital divide. BMJ 2003;326:238.

6. Queluz, THAT. Admissão do Jornal de Pneumologia na SCiELO Brasil: uma vitória com novos desafios. J. Pneumologia [online]. Janeiro/Fevereiro 2002, vol. 28:1

7. Gibbs, W.W. Lost science in the Third World. Sci. Am. 1995; 273:76-83.

8. Beveridge M, Howard A, Burton K, Holder W. The Ptolemy project: a scalable model for delivering health information in Africa. BMJ 2003;327:790-3. 